\title{
Erste nationale Akkreditierung eines interuniversitären Weiterbildungsprogramms im Bereich Gesundheitswesen/Medizin
}

\author{
K. Faisst, B. Holzer
}

Das Interuniversitäre Weiterbildungsprogramm Public Health der Universitäten Basel, Bern und Zürich bietet mit dem Nachdiplomstudium zum Master of Public Health seit 1992 in der Deutschschweiz eine Basisqualifikation für die Weiterbildung zum Facharzt für Prävention und Gesundheitswesen an.

Das Angebot richtet sich aber auch an Personen aus anderen Berufen, die in ihrem gegenwärtigen oder künftigen Berufsalltag mit komplexen Problemen im Gesundheitswesen konfrontiert sind und ihr Qualifikationsprofil diesen Herausforderungen anpassen möchten. Aktuell nutzen 175 Studierende mit einem Hintergrund in Medizin, Natur-, Geistes- und Sozialwissenschaften den Studiengang als Möglichkeit zur Erweiterung ihres beruflichen Spektrums, 114 Personen haben bis Ende des Jahres 2004 den Titel «Master of Public Health» erworben.

\section{Ziel der Ausbildung}

Der interdisziplinäre Ansatz von Public Health ist ein Kernstück der Ausbildung. Ziel ist die Vermittlung von gemeinsamen Perspektiven und Zielen aller im Gesundheitswesen tätigen Personen. Als gemeinsame Basis dienen konzeptionelles Denken, methodische Instrumente, Fachkenntnisse und -kompetenzen, welche in den Disziplinen Epidemiologie, Biostatistik, Gesundheitssoziologie und -psychologie, Health Policy and Health Management sowie in verschiedenen anderen Fächern vermittelt werden. Die strukturellen Eigenheiten des Programms fördern den Austausch und die Netzwerkbildung zwischen Studierenden und aktuellen und künftigen Public-Health-Expertinnen und -Experten.

\section{Zeitlicher Aufwand}

Korrespondenz:

Dr. med. Karin Faisst, MPH MAE Institut für Sozial- und Präventivmedizin

Sumatrastrasse 30

CH-8006 Zürich das gesamte MPH-Curriculum kann je nach persönlichen und beruflichen Ressourcen individuell gestaltet und in 2 bis 5 Jahren berufsbegleitend durchlaufen werden.

\section{Studienaufbau}

Jährlich werden über 30 verschiedene drei- bis zwölftägige Kurse angeboten, die grundsätzlich allen Public-Health-Interessierten aus der Schweiz und dem Ausland offenstehen. Engagierte Dozierende aus dem akademischen und praxisnahen Umfeld sichern einen Ausbildungsstandard auf hohem universitärem Niveau mit Anbindung an die Public-Health-Praxis. Insgesamt dozieren in den Kursen jährlich rund 150 Public-Health-Expertinnen und -Experten aus dem In- und Ausland. Eine begleitete Projektarbeit mit Master-Thesis am Ende des Studiums gibt den Teilnehmenden zudem die Gelegenheit, das Gelernte in die Praxis umzusetzen und so den Erwerb einer professionellen Handlungskompetenz unter Beweis zu stellen.

\section{Qualität des Programms}

Qualitätsmanagement war und ist ein bedeutender integraler Bestandteil des Weiterbildungsprogramms; bereits 2001 war es in einem internationalen Peer-Review-Prozess begutachtet worden.

2004 durchlief der Studiengang als erstes universitäres Nachdiplomstudium im Bereich Gesundheitswesen/Medizin das Akkreditierungsverfahren des Organs für Akkreditierung und Qualitätssicherung (OAQ). Zu diesem Zweck beauftragte das OAQ eine dreiköpfige, internationale Expertengruppe mit der externen Begutachtung. Die unabhängigen Experten, darunter zwei mit ausgewiesener Expertise im Bereich Public Health und mit langjähriger Evaluationserfahrung, hatten die Aufgabe, sowohl die Erfüllung der Qualitätsstandards des OAQs, als auch derjenigen von ASPHER (Association of Schools of 
Public Health in the European Region) zu prüfen. Die Expertengruppe führte dazu Gespräche mit den beiden Programmleiterinnen, Studierenden, Absolventinnen und Absolventen, dem Rektor der Universität Zürich, der Studienleitung und weiteren Mitgliedern des Lehrkörpers an den ISPM in Zürich, Bern und Basel und am Schweizerischen Tropeninstitut (STI) in Basel. Die Experten kamen am Ende einstimmig zur Auffassung, das Programm erfülle die Anforderungen und formulierte Empfehlungen.

Im Schlussbericht des OAQs zuhanden der Schweizerischen Universitätskonferenz SUK streicht das OAQ als besondere Stärken den modularen Aufbau des Programms hervor, der sich bestens zum berufsbegleitenden Studium eignet, sowie die ausgezeichneten Kontaktmöglichkeiten mit der Public-Health-Berufsgemeinschaft in der Schweiz. Auch wird dem Weiterbildungsprogramm ein grosses Potential für eine internationale Entwicklung im europäischen Weiterbildungsangebot zugesprochen.

Ende Februar 2005 hat nun die Schweizerische Universitätskonferenz das Akkreditierungsgesuch gutgeheissen. Die Akkreditierung wurde an die Auflage gebunden, dass das Deutschschweizer MPH-Programm in die zu realisierende «Swiss School of Public Health» integriert wird, mit dem Ziel, alle nationalen Studiengänge von Public Health und Gesundheitsökonomie unter einem Dach zu vereinen und zu stärken.
Als Zeichen der erfolgten Akkreditierung wird ein Qualitätszertifikat inkl. Qualitätssiegel ausgehändigt, das dem Programm das Erreichen der international ausgerichteten Qualitätsstandards bescheinigt.

\section{Künftige Herausforderungen}

Inhaltlich und strukturell wird das Programm weiterhin laufend an internationale Entwicklungen angepasst. Hier steht im Vordergrund, die Empfehlungen der Experten zur Qualitätsverbesserung umzusetzen und die Ausbildung noch mehr an Problemlösungen und an den in der Praxis benötigten Kompetenzen zu orientieren.

Im Mittelpunkt der nächsten Jahre steht die 2005 zu gründende Swiss School of Public Health (SSPH+). Die Etablierung dieser Postgraduiertenschule wird die Professionalisierung und Weiterentwicklung des Deutschschweizer MPH-Programms auf allen Ebenen sowie den Ausbau nationaler und internationaler Kooperationen mit themenverwandten Weiterbildungsprogrammen unter Nutzung der gemeinsamen Schnittstellen unterstützen.

Mehr Informationen zum Interuniversitären Weiterbildungsprogramm Public Health unter www.public-health-edu.ch oder direkt bei der Koordinationsstelle Public Health, Sumatrastrasse 30, 8006 Zürich, Tel. 0446344651. 